\title{
Rainbow Particle Imaging Velocimetry for Dense 3D Fluid Velocity Imaging
}

\author{
JINHUI XIONG, RAMZI IDOUGHI, ANDRES A. AGUIRRE-PABLO, ABDULRAHMAN B. ALJEDAANI, \\ XIONG DUN, QIANG FU, SIGURDUR T. THORODDSEN, and WOLFGANG HEIDRICH,
}

King Abdullah University Of Science And Technology
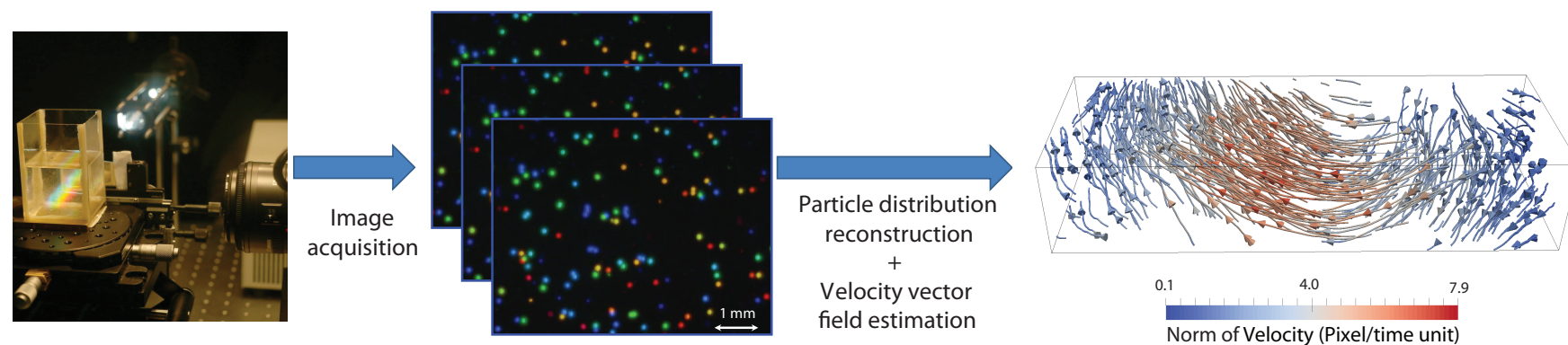

Fig. 1. Using a rainbow color-coded PIV setup in combination with a hybrid diffractive/refractive camera optics(left) we can encode 3D particle positions in fluid flows into a single camera image, while keeping all particles in focus simultaneously (center). From a sequence of such images, the 3D particle positions and the dense fluid vector field can be reconstructed using an optimization-based approach. The vector field is represented as a regular grid covering the entire flow volume, and can be visualized by showing the path lines of new (synthetic) particles (right)

Despite significant recent progress, dense, time-resolved imaging of complex, non-stationary 3D flow velocities remains an elusive goal. In this work we tackle this problem by extending an established 2D method, Particle Imaging Velocimetry, to three dimensions by encoding depth into color. The encoding is achieved by illuminating the flow volume with a continuum of light planes (a "rainbow"), such that each depth corresponds to a specific wavelength of light. A diffractive component in the camera optics ensures that all planes are in focus simultaneously. With this setup, a single color camera is sufficient for tracking $3 \mathrm{D}$ trajectories of particles by combining $2 \mathrm{D}$ spatial and $1 \mathrm{D}$ color information.

For reconstruction, we derive an image formation model for recovering stationary 3D particle positions. 3D velocity estimation is achieved with a variant of 3D optical flow that accounts for both physical constraints as well as the rainbow image formation model. We evaluate our method with both simulations and an experimental prototype setup.

CCS Concepts: • Computing methodologies $\rightarrow$ Computer graphics; $3 D$ imaging; Motion capture;

Additional Key Words and Phrases: Fluid velocity imaging, Rainbow PIV, Optimization

This work was supported by a KAUST CRG3 research grant as well as KAUST baseline funding.

Address: King Abdullah University of Science and Technology Al Khwarizmi Bldg 1 , Rm 2113, Thuwal, 23955-6900, Kingdom of Saudi Arabia

Email: <firstname>.<lastname>@kaust.edu.sa.

Permission to make digital or hard copies of all or part of this work for personal or classroom use is granted without fee provided that copies are not made or distributed for profit or commercial advantage and that copies bear this notice and the full citation on the first page. Copyrights for components of this work owned by others than ACM must be honored. Abstracting with credit is permitted. To copy otherwise, or republish, to post on servers or to redistribute to lists, requires prior specific permission and/or a fee. Request permissions from permissions@acm.org.

(c) 2017 ACM. 0730-0301/2017/7-ART36 \$15.00

DOI: http://dx.doi.org/10.1145/3072959.3073662

\section{ACM Reference format:}

Jinhui Xiong, Ramzi Idoughi, Andres A. Aguirre-Pablo, Abdulrahman B. Aljedaani, Xiong Dun, Qiang Fu, Sigurdur T. Thoroddsen, and Wolfgang Heidrich. 2017. Rainbow Particle Imaging Velocimetry for Dense 3D Fluid Velocity Imaging. ACM Trans. Graph. 36, 4, Article 36 (July 2017), 14 pages. DOI: http://dx.doi.org/10.1145/3072959.3073662

\section{INTRODUCTION}

Fluid capture is an active research area in computer graphics. Recent works include efforts to image phenomena such as flames [Hasinoff and Kutulakos 2007; Ihrke and Magnor 2004], smoke [Gu et al. 2013; Hawkins et al. 2005], transparent hot air flows [Atcheson et al. 2008], and fluid mixtures [Gregson et al. 2012]. While these methods recover dense volumetric reconstructions, they only yield independent scalar fields of the density of the respective phenomenon at each time step. To fully characterize the 3D flow and open up applications beyond simple play-back, 3D velocity fields need to be recovered as well. While there have been efforts to recover velocities from the captured scalar fields through optical flow-style approaches, these attempts have been limited by the relatively small amount of high-frequency texture in the recovered data [Gregson et al. 2014].

Fluid imaging has many significant applications in scientific and engineering fields such as combustion research, design of airplanes and underwater vehicles, and development of artificial heart valves. Since 3D unsteady flows and turbulence are very common in such domains, the main task of the fluid imaging is to allow probing the fluid motions over a range of length scales. In other words, the ultimate goal is to be able to obtain 3D dense measurements of the three components of the velocity vector, known as 3D-3C. 
Over the last decades, different imaging techniques have been developed to get closer to this goal. Particle Imaging Velocimetry (PIV) is the most commonly used of these techniques [Adrian and Westerweel 2011; Lourenco et al. 1989]. For PIV, small density-matched tracer particles are inserted into the flow, and their advected motion is tracked with image correlation methods, i.e. optical flow. In basic 2D PIV [Okamoto et al. 2000], this tracking is made possible by illuminating the volume with a light sheet perpendicular to the camera line of sight (Fig. 2, left). Particles within that plane can be identified easily, and tracked over time, so long as the flow does not move them out of plane. This yields dense measurements of two components of the velocity field on a two-dimensional slice of the volume (2D-2C). Although 3D extensions such as holographic PIV [Hinsch 2002] or tomographic PIV [Elsinga et al. 2006] exist, a dense reconstruction of all three components of the velocity field over the full 3D volume requires multiple cameras and remains elusive in practice (also see Section 2). The densest volume measurements involve high-speed imaging in combination with scanning laser-volumes [Casey et al. 2013].

This paper proposes a new approach, RainbowPIV, by combining a suitable setup for color-based encoding of the third dimension in volumetric PIV, as well as a powerful algorithm to retrieve both the particle positions and the velocity vector field. For the hardware part, a linear color filter is employed in order to obtain a continuous wavelength-gradation pattern, i.e. a rainbow illumination. Then, a diffractive optical element (DOE) is attached to the camera objective lens, in order to achieve a wavelength-selective focus that coincides with the rainbow illumination planes (Fig. 2, right). With this setup, particles with different wavelengths (different depths) will be simultaneously in focus on the sensor plane.

The reconstruction algorithm utilizes a detailed image formation model for this setup to retrieve the 3D location of particles in each frame. From a sequence of successive frames, the velocity vector field is reconstructed using an optical flow approach [Horn and Schunck 1981; Meinhardt-Llopis et al. 2013], where physical constraints (incompressibility and temporal consistency) are introduced. In order to improve the obtained results, we can iterate between position and velocity estimation, effectively solving a joint optimization problem for both. The specific contributions of this paper are:

- We propose a simple PIV setup (RainbowPIV) for measuring time-varying 3D-3C fluid velocity vector fields using a single camera.

- We design a hybrid refractive-diffractive optical system in order to focus all wavelength on the same sensor plane, extending the depth-of-field while preserving high lateral resolution.

- We formulate an image formation model for 3D particle distribution reconstruction, and apply optimization strategies to tackle the ill-posed inverse problem.

- We introduce a physically constrained optical flow method for recovering the fluid velocity fields, and evaluate its effectiveness on synthetic data. Our approach allows having a good estimation of velocity over the measurement volume (high concentration of particles).
- We demonstrate our proposed hardware setup and algorithms on real fluid flows.

\section{RELATED WORK}

3D Fluid Imaging in Graphics has, as already mentioned, mostly focused on independent scalar density fields for each time step. Examples for the physical properties recovered in this fashion include the distribution of light emission in flames [Hasinoff and Kutulakos 2007; Ihrke and Magnor 2004], scattering density in smoke [Gu et al. 2013; Hawkins et al. 2005], density of a fluorescent dye in fluid mixtures [Gregson et al. 2012], as well as the refractive index distribution in hot air plumes [Atcheson et al. 2008]. While this data is sufficient for playback in graphics applications, other interesting applications such as guided simulation [Wang et al. 2009], or flow editing require velocity fields instead of just scalar densities. This requires some form of velocity estimation or flow tracking, which is difficult on this kind of input data (see below).

3D Particle Reconstruction is an alternative to imaging continuous densities, and is used by $3 \mathrm{D}$ variants of PIV. The task of the particle reconstruction is to determine the 3D location of particles from one or more camera views. The total number of cameras in these settings is usually very limited due to space constraints, as well as occlusions by solids, and is typically orders of magnitude lower than the number of projections in x-ray tomography, for example. Another practical issue is depth of field of the cameras, since the whole volume needs to be in focus simultaneously, and the camera aperture usually has to be large to collect enough light to capture fast flows.

Some examples of 3D extensions of PIV include holographic PIV [Hinsch 2002], which works with coherent light, and tomographic PIV [Elsinga et al. 2006; Schanz et al. 2016], which utilizes typically 3-5 cameras. Both of these approaches are in practice very hard to set up for many types of flow experiments.

More closely related to our approach are single-camera methods, with drastically simplified setup. Willert et al. [1992] used a three-pin-hole mask to decode illuminated particles such that the three-dimensional positions of each particle can be retrieved from the image patterns on the observed image via a defocus analysis. Since three dots would appear in the image for each particle, this method is stuck with a low particle seeding density. Another group of approaches made use of plenoptic cameras [Levoy et al. 2006; $\mathrm{Ng}$ et al. 2005], which capture the full 4D light field. Particle positions can be reconstructed using ray tracing based algorithms. The idea of applying such technology for measuring volumetric particle distributions has been discussed by [Lynch et al. 2012]. However, due to the existence of ghost particles originated from reconstruction algorithm and reduced spatial resolution, it becomes difficult to reveal particle locations with relatively high accuracy. This can also be seen as limited angle tomography with a very narrow cone of observations.

Instead of modifying the camera side, another class of volumetric particle reconstruction approaches relies on modifying the illumination method, providing additional information on the relative depth, as seen from the camera, by encoding it in color. For this purpose different illumination methods were used: prism [Kimura et al. 

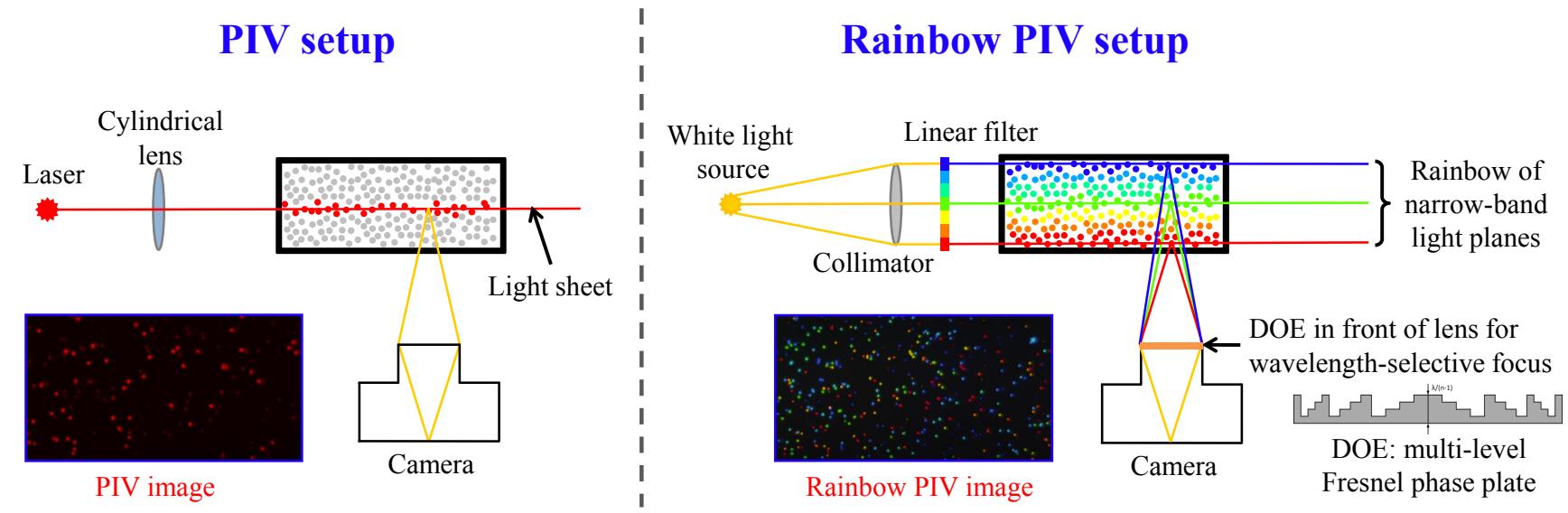

Fig. 2. Comparison between the measurement setups for standard PIV (left) and RainbowPIV (right). In regular PIV, a single plane of the volume is illuminated by a light sheet, and a camera focusing on this plane is able to track particles moving within it. This yields 2 components of the velocity field on a $2 \mathrm{D}$ slice of the volume. By comparison, in RainbowPIV, the 3D volume is illuminated by a continuum of planes, where the wavelength of the illumination varies linearly with the depth. Particles in the volume can thus be modeled as narrow-band point sources, and a diffractive optical element attached to the camera lens ensures that for each wavelength the camera is focused at the appropriate depth.

1991], laser [McGregor et al. 2007], color filter [Pick and Lehmann 2009], LCD projector [Watamura et al. 2013]. Herein, the locations of the particles in the volume can be determined by their 2D spatial positions and by the colors in the captured image using a mapping between color and depth position. The primary advantage of this setup compared to other 3D PIV methods is its simplicity. Nevertheless, the presence of random noise, optical aberrations and focus issues, color contamination caused by secondary light scattering from the particles, and color mixing for overlapping particles severely complicate the identification of the representative colors for every possible particle in the observed image. McGregor et al. [2007] used a method based on a calibration curve relating the hue of acquired images to the depth of particles within the imaged volume. Watamura et al. [2013] proposed an algorithm to calculate particle's representative color by averaging hue values of the pixels where the particle is projected on in polar coordinate. Even though it revealed promising results for Particle Tracking Velocimetry (PTV), where low density particles are seeded, it will fail for our task of measuring a dense set of velocity fields, where sufficiently high density of particles is required.

Our method exploits a similar idea of encoding particle depth by color, however we employ a combination of coded illumination and modified camera optics to solve many of the issues of existing methods. Moreover, we develop a new optimization-based joint reconstruction of both particle position and velocity field.

Velocity Estimation from particle fields has been elaborately studied not only in the field of fluid mechanics, but also in the computer vision community. Literature from the fluid mechanics field mainly adopts correlation-based algorithms [Prasad 2000] for global velocity measurement, which computes the spatial auto-correlation or cross-correlation of successive images, extracting average motion at every single interrogation spot. Though significant improvements have been made on correlation methods [Stanislas et al. 2008], they still have issues in areas of low particle density, which is common in $3 \mathrm{D}$ measurements.

In a seminal result from computer vision, Horn and Schunck [1981], proposed a global variational optical flow method based on the assumption of brightness constancy and smoothness in the flow vector. The connection between optical flow and fluid flow was investigated by Liu and Shen [2008], which revealed that under certain conditions (mass conservation, inviscid), brightness constancy is equivalent to scalar transport equation for fluid flow. This connection lends a physical meaning to optical flow approaches for fluid tracking. Heitz et al. [2010] gave an overview to the applications of optical flow based fluid motion estimation. The estimation accuracy between optical flow and correlation approaches applied to PIV system has been numerically evaluated [Liu et al. 2015].

Since the optical flow problem is physically connected to the continuity equation in fluid dynamics, it becomes feasible to introduce Navier-Stokes equations, which govern real-world fluid motions, as additional physical priors into the conventional Horn-Schunck algorithm. Some previous literature has taken divergence-free constraints into account [Herlin et al. 2012; Ruhnau et al. 2007; Yuan et al. 2007], while most of them suffer from the complexity of solving higher order regularization terms. Gregson et al. [2014] simplify this issue by connecting the pressure projection method with the proximal operator, allowing it to be easily handled by a convex optimization framework. Ruhnau et al. [2007] and Heitz et al. [2008] also consider the equation for time evolution of fluid flow, imposing temporal consistency. In our work, we adopt ideas from fluid simulation [Fedkiw et al. 2001; Foster and Metaxas 1997; Stam 1999], which approximately solve the time-evolution of fluid flow. This enables us to integrate the temporal coherence regularization terms into the optical flow model, which can then be solved by a modular optimization framework. 


\section{RAINBOW PARTICLE IMAGING VELOCIMETRY}

The RainbowPIV method consists of two components: a new optical setup that encodes particle depth into a color image with a large depth of field, and a matching new reconstruction algorithm that jointly optimizes particle position and velocity field.

Optical Setup. A comparison between the setups for regular PIV and RainbowPIV is shown in Figure 2. In regular PIV (left), a laser is used to create a light sheet perpendicular to the camera line of sight. The camera is focused on the illuminated plane, and can therefore observe and track particles moving within the sheet of light. This yields 2 components of the velocity field on a $2 \mathrm{D}$ slice of the volume (2D-2C). In RainbowPIV, the illumination is provided by a white light source that is collimated and filtered so that the wavelength varies linearly with the depth within the flow volume. In this setup, particles submersed in the fluid can be modeled as narrow-band point lights, whose wavelength varies linearly with depth.

The second part of the optical setup is a diffractive optical element in the form of a Fresnel phase plate, which is implemented as a thin glass plate with a micro-scale height field structure etched into it. This DOE provides a wavelength-selective focus in the camera optics. Specifically, the optical system is designed such that the camera focus for each wavelength corresponds to the depth at which that wavelength occurs in the rainbow illumination. This design achieves all-in-focus imaging of the particles in the interrogation volume.

Reconstruction. The reconstruction task is to estimate particle positions from the the observed color, and then track these particles over time to obtain a 3D velocity field to get a full 3D, 3 component (3D-3C) measurement. This task is made more complicated by the fact that the camera captures only RGB information, not a full hyperspectral image, which makes the position reconstruction less robust. To tackle this problem, we employ an iterative approach: an initial position estimate for each time step can be used to obtain a first estimate of the time-dependent velocity field. This velocity field can be used to refine the position estimate by adding physical priors that tie together all the time steps. These two iterative steps are described in detail below. For tomoPIV a joint reconstruction of volume and velocity was already used by [Barbu et al. 2013].

\subsection{Particle Position Estimation}

An inverse problem is proposed for recovering particle locations in 3D spatial domain. We start by introducing an image formation model that relates the obtained particle positions to the observed image. Three regularization terms are then added to formulate an optimization problem, which can be efficiently solved with guaranteed convergence, tackling our ill-posed inverse problem.

Image Formation Model. As mentioned above, the illumination in the volume is designed to consist of a continuum of light sheets (Figure 2) with a narrow-band spectrum, whose wavelength (denoted as $\lambda$ ) varies with depth ( $z$ coordinate). In this work, we restrict ourselves to a linear relationship between $z$ and $\lambda$ since this setting is easily implemented with off-the-shelf components (see Section 4). Therefore, the location of particles in the volume can be geometrically represented as the position of the light plane, specified by wavelength, and pixel positions in that light plane $(\mathbf{x}, \lambda)=(x, y, \lambda)$
The presence of a particle at a specific point in the volume is modeled as an occupancy probability $P(\mathbf{x}, \lambda)$.

Since we are operating with incoherent light, the imaging process of the optical system can be modeled as a set of point spread functions (PSF), one for each color channel: $g_{C}(\mathbf{x}, \lambda)$, where $C \in$ \{red, green, blue\}. With these definitions, the image formation model is

$$
i_{C}(\mathbf{x})=\int_{\Lambda} \int_{X} g_{C}\left(\mathbf{x}-\mathbf{x}^{\prime}, \lambda\right) \cdot i_{r}(\mathbf{x}, \lambda) \cdot P(\mathbf{x}, \lambda) d \mathbf{x}^{\prime} d \lambda,
$$

where $i_{C}(\mathbf{x})$ are the color channels of the captured RGB image, and $i_{r}(\mathbf{x}, \lambda)$ is the corresponding spectral distribution incident on the image sensor. The spatial integral corresponds to a convolution representing potentially imperfect focus, while the wavelength integral represents the conversion from a spectral image to an RGB image encoding 3D particle positions.

Optimization Problem. After discretization, we can formulate the convolution of PSFs and reflected light intensity as a matrix A $\in \mathbb{R}^{3 N \times N L}$, where $N$ is the number of image pixels, $L$ is the number of discretization levels along the wavelength coordinate, and the value of 3 refers to three color channels. Moreover, $\mathbf{i}_{t} \in \mathbb{R}^{3 N}$ represents the observed image at time $t$, and $\mathrm{p}_{t} \in[0,1]^{N L}$ is the volume of occupancy probabilities at time $t$. The distribution of particles at each time step of a video can be retrieved by solving the linear system

$$
\operatorname{Ap}_{t}=\mathbf{i}_{t} .
$$

However, this inverse problem is ill-posed as we have compressed the full spectral information encoding the particle position into just three color channels. To handle this ill-conditioned inverse problem, some prior knowledge of the distribution of particles is introduced as regularization terms, resulting the following minimization problem:

$$
\begin{aligned}
\left(\mathbf{p}^{*}\right) & =\underset{\mathbf{p}}{\operatorname{argmin}} \frac{1}{2}\left\|\mathbf{A}\left[\mathbf{p}_{1}|\ldots| \mathbf{p}_{T}\right]-\left[\mathbf{i}_{1}|\ldots| \mathbf{i}_{T}\right]\right\|_{2}^{2} \\
& +\kappa_{1}\left\|\operatorname{diag}(\mathbf{w})\left(\mathbf{p}_{1} ; \ldots ; \mathbf{p}_{T}\right)\right\|_{1}+\Pi_{[0,1]}\left(\mathbf{p}_{1} ; \ldots ; \mathbf{p}_{T}\right) \\
& +\kappa_{2} \sum_{t=1}^{T} \int_{\Omega} \mathbf{p}_{t} \odot\left(\mathbf{p}_{t}-\mathbf{p}_{t+1}\left(\mathbf{u}_{t},-\Delta t\right)\right)^{\circ 2} d \Omega,
\end{aligned}
$$

where $\odot$ and $(\cdot)^{\circ 2}$ respectively refer to the operators for the Hadamard (i.e. component-wise) product and square operator. The operator $\Pi_{[0,1]}$ projects all volume occupancy probabilities onto the convex set of valid probabilities $[0,1]^{N L}$.

The first line in Equation 3 is a least-square data fitting term corresponding to Equation 2. The second line defines a weighted $L_{1}$ term that encourages sparse distributions of particles in the volume, and the indicator function enforces that occupancy probabilities are between zero and one. Finally, the term of the third line provides temporal coherence by mandating that occupancy probabilities of successive time frames are consistent with advection under a previously estimated flow field $\mathbf{u}_{\mathbf{t}}=\left(u_{t}, v_{t}, w_{t}\right)$ by $-\Delta t$ units of time, expressed as $\mathrm{p}_{t+1}\left(\mathbf{u}_{t},-\Delta t\right)$. We call this term the particle motion consistency term, and it allows for refining position estimates once a velocity field has been estimated, and ties the reconstruction of 
all frames together into a single optimization problem. The particle motion consistency term is discussed in more detail below.

The above optimization problem is non-smooth because of the $L_{1}$ term and the indicator function, hence it cannot be solved by general optimization tools such as gradient descent. The strategy tackling this kind of issue is to decouple non-smooth terms from the original optimization problem, such that distinct parts can be handled separately. We apply this strategy using the ADMM framework which is systematically discussed in [Boyd et al. 2011].

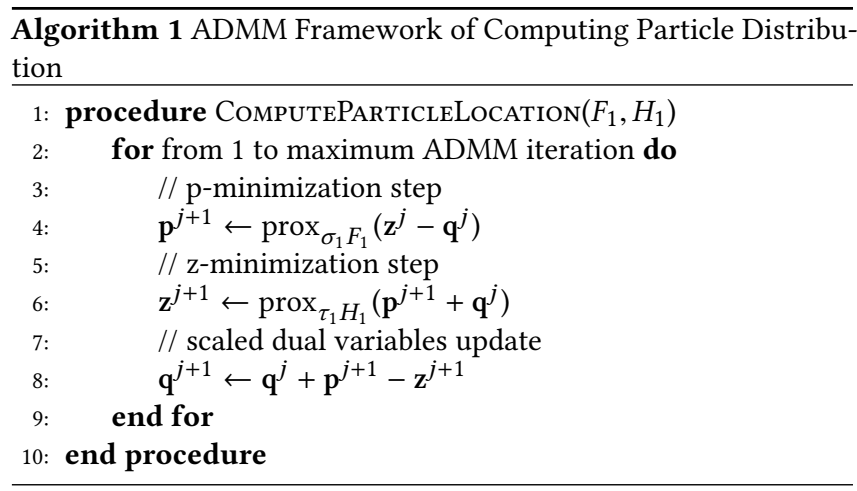

The pseudo code for solving Equation 3 using ADMM is shown in Algorithm 1, where $j$ is the iteration number, $\mathbf{z}$ is a slack variable, $\mathbf{q}$ is a dual variable, or Lagrange multiplier. prox ${ }_{\sigma_{1} F_{1}}$ and $\operatorname{prox}_{\tau_{1} H_{1}}$ are proximal operators [Parikh et al. 2014] based on $F_{1}$ and $H_{1}$ respectively, and we provide their derivations in the Appendix. $F_{1}$ and $H_{1}$ are defined as:

$$
\begin{aligned}
F_{1}(\mathbf{p}) & =\frac{1}{2}\left\|\mathbf{A}\left[\mathbf{p}_{1}|\ldots| \mathbf{p}_{T}\right]-\left[\mathbf{i}_{1}|\ldots| \mathbf{i}_{T}\right]\right\|_{2}^{2} \\
& +\kappa_{2} \sum_{t=1}^{T} \int_{\Omega} \mathbf{p}_{t} \odot\left(\mathbf{p}_{t}-\mathbf{p}_{t+1}\left(\mathbf{u}_{t},-\Delta t\right)\right)^{\circ 2} d \Omega \\
H_{1}(\mathbf{p}) & =\kappa_{1}\left\|\operatorname{diag}(\mathbf{w})\left(\mathbf{p}_{1} ; \ldots ; \mathbf{p}_{T}\right)\right\|_{1}+\Pi_{[0,1]}\left(\mathbf{p}_{1} ; \ldots ; \mathbf{p}_{T}\right)
\end{aligned}
$$

Particle Sparsity. The $L_{1}$ penalized term ensures a sparse distribution of particles in the volume. It is further weighted by a diagonal matrix $\operatorname{diag}(\mathbf{w})$. Unlike the algorithm proposed in [Candes et al 2008], which iteratively changes the weight coefficients based on previous results for enhancing sparsity, weights in our approach are fixed during iterations, but vary with particle depth. The motivation for this process is to compensate for different sensitivities of the camera to different wavelengths. For example, wavelengths in the yellow or in the blue-green part of the spectrum elicit a strong response in two or even three color channels, while wavelengths in the far blue or far red parts only trigger one channel. This can result in a non-uniform particle distribution, where particles are more likely to be placed at certain preferred depths. The weighting term allows us to eliminate this bias by compensating for the photometric non-uniformity.

Particle Motion Consistency. As mentioned, particle motion consistency ensures that estimated particle locations in successive frames are consistent with advection through a previously estimated flow field. This turns the position estimation from a set of independent problems, one for each time step, to a single joint estimation problem for the whole sequence. This term can be improved by adding a mask to suppress the impact of low confidence flow estimates.

\subsection{Velocity Field Reconstruction}

This section describes how we estimate the fluid flow vectors from reconstructed 3D particle distributions in a video frame. First, we introduce the physical properties of fluid flow formulated in NavierStokes equations, and then an optimization problem is constructed by combining conventional optical flow with those physical constraints.

Divergence Free. An incompressible flow can be described as a solenoidal flow vector field $\mathbf{u}_{\text {sol }}$, which is divergence free:

$$
\nabla \cdot \mathbf{u}_{\text {sol }}=0 .
$$

Based on the Helmholtz decomposition, any arbitrary vector field $\mathbf{u}$ (in our case an intermediate flow vector that does not necessarily satisfy the divergence-free constraints) can be decomposed into a solenoidal (divergence-free) part and an irrotational (curl-free) part. The irrotational flow vector is the gradient of some scalar function (pressure $\mathcal{P}$ in our case), hence we can express the Helmholtz decomposition as

$$
\mathbf{u}=\mathbf{u}_{\text {sol }}+\nabla \mathcal{P} / \rho
$$

where $\rho$ defines density. Taking the divergence of both sides, we obtain

$$
\nabla \cdot \mathbf{u}=\nabla^{2} \mathcal{P} / \rho\left(\text { since } \nabla \cdot \mathbf{u}_{\text {sol }}=0\right) .
$$

With the intermediate vector field $\mathbf{u}$, the scalar function $\mathcal{P}$ can be computed by solving the above Poisson equation, and then the solenoidal flow vector field can be simply retrieved as

$$
\mathbf{u}_{\text {sol }}=\mathbf{u}-\nabla \mathcal{P} / \rho \text {. }
$$

Equations 8 and 9 represent a pressure projection $\Pi_{C_{D I V}}$ operation that projects an arbitrary flow field onto the space of divergence-free flows $C_{D I V}$, and is widely used in fluid simulation. Mathematically, this step corresponds to an operator splitting method [Gregson et al. 2014].

Temporal Coherence. The incompressible Navier-Stokes equation describes the time evolution of fluid velocity vector fields, given by:

$$
\frac{\partial \mathbf{u}}{\partial t}+(\mathbf{u} \cdot \nabla) \mathbf{u}=-\nabla \mathcal{P} / \rho+(\nabla \cdot \overline{\bar{\tau}}) / \rho+f,
$$

where $\mathcal{P}$ is the pressure, $\overline{\bar{\tau}}$ is deviatoric stress and $f$ is an external force. For a non-viscous fluid in absence of external force and ignoring the unknown pressure gradient term, Equation 10 becomes

$$
\frac{\partial \mathbf{u}}{\partial t}+(\mathbf{u} \cdot \nabla) \mathbf{u}=0
$$

which refers to an approximated evolution of fluid velocity over time. On the basis of this equation, we can advect the fluid velocity at the current time step by itself, and then project it onto a space of divergence-free flows to generate an estimate of the subsequent velocity field, and vice versa. This time evolution equation will be 
introduced into the optimization problem discussed in the following as a soft constraint.

Optimization Problem. We aim to reconstruct the fluid flow velocity vector fields based on a physically constrained optical flow model. The extended optical flow model is formulated as:

$$
\begin{aligned}
\mathbf{u}_{t}^{*}=\underset{\mathbf{u}_{t}}{\operatorname{argmin}} & \int_{\Omega} \mathbf{p}_{t} \odot\left(\mathbf{p}_{t}-\mathbf{p}_{t+1}\left(\mathbf{u}_{t},-\Delta t\right)\right)^{\circ 2} d \Omega+\kappa_{3}\left\|\nabla \mathbf{u}_{t}\right\|_{2}^{2} \\
& +\kappa_{4}\left(\left\|\mathbf{M}\left(\mathbf{u}_{t}-\Pi_{C_{D I V}}\left(\mathbf{u}_{t-1}\left(\mathbf{u}_{t-1}, \Delta t\right)\right)\right)\right\|_{2}^{2}\right. \\
& \left.+\left\|\mathbf{M}\left(\mathbf{u}_{t}-\Pi_{C_{D I V}}\left(\mathbf{u}_{t+1}\left(\mathbf{u}_{t},-\Delta t\right)\right)\right)\right\|_{2}^{2}\right) \\
& +\Pi_{C_{D I V}}\left(\mathbf{u}_{t}\right),
\end{aligned}
$$

each line of which is explained hereafter:

- the first line describes the conventional Horn-Schunck optical flow model except that the brightness constancy constraint is replaced with the masked particle motion consistency as discussed in Section 3.1.

- the second and third lines describe the temporal coherence regularization as explained above: the fluid velocity at the current time step is approximated by either forward warping the flow vector at the previous time step by itself, followed by a projection operation, or by backward warping the flow vector at the next time step by the current flow, followed again by a projection operation. The binary mask $\mathbf{M}$ is employed to ensure confidence-based weighting, giving 0 for the flow vectors near the boundary and 1 for vectors in the central region.

- the fourth line represents an indicator function of the projection method introduced above. Gregson et al. [2014] found that the projection operation is equivalent to the proximal operator for the space of divergence-free velocity field. This allows us to integrate the divergence-free constraint into the original optical flow model, which can still be efficiently solved by well-known optimization frameworks.

We formulate this optimization problem in the ADMM framework in Algorithm 2, where the definitions of the functions $F_{2}$ and $H_{2}$ are given below. The corresponding proximal operators can be found in the Appendix.

$$
\begin{aligned}
F_{2}\left(\mathbf{u}_{\mathbf{t}}\right) & =\int_{\Omega} \mathbf{p}_{t} \odot\left(\mathbf{p}_{t}-\mathbf{p}_{t+1}\left(\mathbf{u}_{t},-\Delta t\right)\right)^{\circ 2} d \Omega+\kappa_{3}\left\|\nabla \mathbf{u}_{\mathbf{t}}\right\|_{2}^{2} \\
& +\kappa_{4}\left(\left\|\mathbf{M}\left(\mathbf{u}_{\mathbf{t}}-\Pi_{C_{D I V}}\left(\mathbf{u}_{t-1}\left(\mathbf{u}_{t-1}, \Delta t\right)\right)\right)\right\|_{2}^{2}\right. \\
& \left.+\left\|\mathbf{M}\left(\mathbf{u}_{\mathbf{t}}-\Pi_{C_{D I V}}\left(\mathbf{u}_{t+1}\left(\mathbf{u}_{t},-\Delta t\right)\right)\right)\right\|_{2}^{2}\right) \\
H_{2}\left(\mathbf{u}_{\mathbf{t}}\right) & =\Pi_{C_{D I V}}\left(\mathbf{u}_{\mathbf{t}}\right)
\end{aligned}
$$

In addition, a coarse-to-fine strategy is applied to deal with large displacements. The algorithm begins from the coarsest level, and an initial guess of optical flow at the next finer level is obtained by scaling up the flow computed in the coarser level. It should be noted that in this case, the above optimization problem becomes non-linear in $\mathbf{u}_{t}$ on account of the warping term $\mathrm{p}_{t+1}\left(\mathbf{u}_{t},-\Delta t\right)$. To

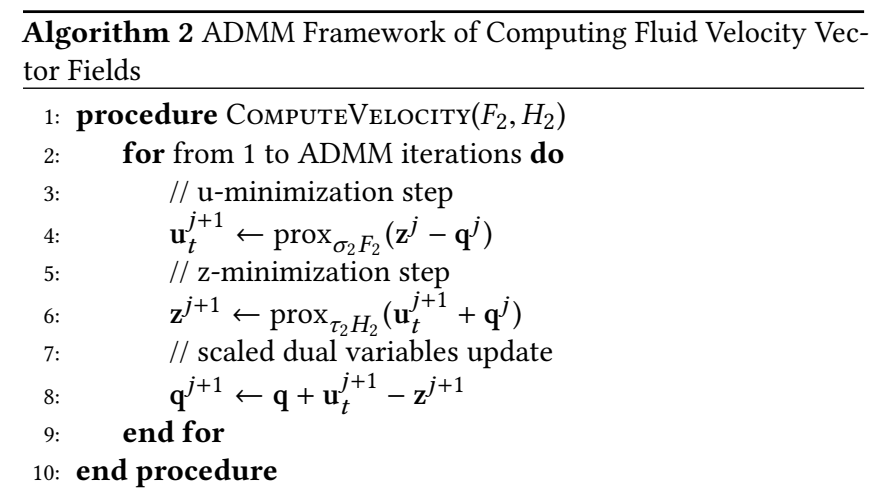

tackle this issue, the non-linear term is linearized by using first order Taylor expansion and $\mathbf{u}_{t}$ is updated iteratively based on fixed-point theorem. More detailed descriptions about this approach are given in [Meinhardt-Llopis et al. 2013].

For a sequence of fluid velocity vector fields, each of them is solved independently in an iterative loop. The update of the flow at one time step will impact the subsequent flows in current iteration, and also the previous flows in the following iterations.

\section{RESULTS AND DISCUSSION}

\subsection{Experimental setup}

Figure 3 represents a picture of the experimental configuration used to evaluate the performance of the RainbowPIV algorithm.

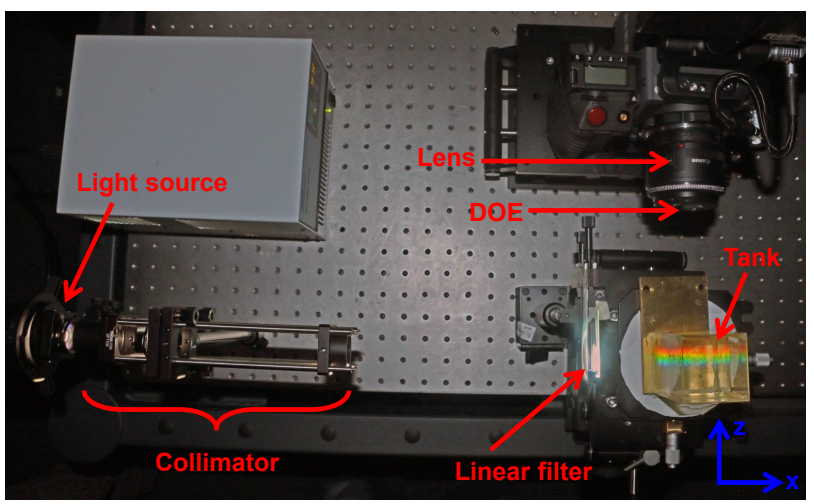

Fig. 3. Illustration of the experimental setup. A combination of a white light source, a collimator and a linear bandpass filter yields a parallel rainbow beam. After reflection on the particles present in the tank, the light is acquired by a camera. A hybrid refractive-diffractive lens (lens+DOE) is used to ensure that all particles of the measurement volume are focused on the same sensor plane.

Rainbow light generation. The experiments were performed using a high power plasma light source combined with a liquid light guide (HPLS245, Thorlabs) to generate white light (output spectrum: [390, $730 \mathrm{~nm}]$ ). A collimator was added to obtain a parallel light beam. It is important to have a parallel light beam, to guarantee that two particles having the same depth will be illuminated by the same colored light. 
To split the white light in a rainbow beam, we employed a continuously linearly varying bandpass filter (LF103245, Delta Optical Thin Film). Other components (prism, blaze grating) were also considered for their ability to generate a rainbow beam. However after comparison, the linear filter appeared to us as the best solution for its effectiveness and simplicity. The generated beam encompasses a spectral range from $480 \mathrm{~nm}$ to $680 \mathrm{~nm}$, and corresponding to a depth range of $18 \mathrm{~mm}$ in the $z$ direction. Given the height of the beam and the length of the used tank, the two other dimensions of the measurement volume are $50.1 \mathrm{~mm}$ along the $x$ axis and $25.6 \mathrm{~mm}$ along the $y$ axis.

Acquisition device. To record the particle images, a digital camera was used (RED SCARLET-X DSMC, sensor: MYSTERIUM-X [30 $\mathrm{mm} \times 15 \mathrm{~mm}$ ], $4096 \times 2160$ pixels). A lens with a focal length of $50 \mathrm{~mm}$ was mounted on the camera. As can be seen in Figure 5 (b, c, d), when a standard refractive lens is used alone, the depth of field is very shallow, and only a small depth range can be in focus. For these three cases the other wavelengths are out of focus, which makes it impossible to exploit these images to retrieve the velocity of particles.

A DOE (see Figure 4) was designed in order to overcome this limitation. Specifically, the DOE is a Fresnel phase plate, which is implemented as a height field with 16 discrete levels as discussed in other recent works, e.g. [Heide et al. 2016; Peng et al. 2015]. The design parameters for the hybrid refractive/diffractive camera lens are summarized in the following table (please refer to the Appendix for a description on how to derive these parameters):

\begin{tabular}{|l|l|l|}
\hline Symbol & Description & Value \\
\hline$D_{D O E}$ & DOE diameter & $16 \mathrm{~mm}$ \\
$f_{\lambda_{0}}^{D O E}$ & DOE focal length for $\lambda_{0}=563 \mathrm{~nm}$ & $401.8 \mathrm{~mm}$ \\
$\gamma$ & Magnification & 2.065 \\
$F \#$ & Aperture & 4.125 \\
$L^{\prime}$ & Distance hybrid lens - sensor & $66 \mathrm{~mm}$ \\
$L_{2}$ & Distance hybrid lens - volume & $127.3 \mathrm{~mm}$ \\
\hline
\end{tabular}

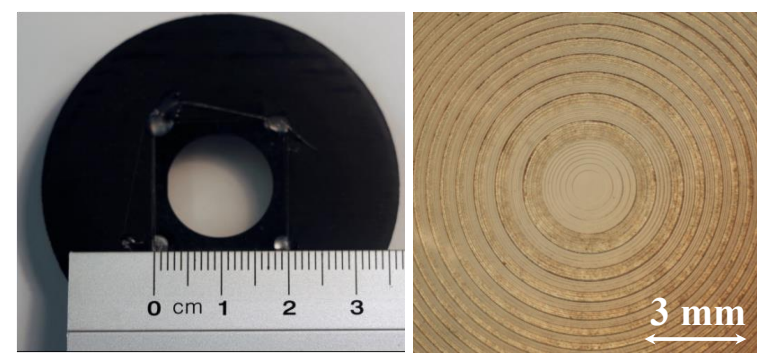

Fig. 4. Left: The designed DOE fixed on a support, and destined to be mounted on the refractive lens. Right: A microscopic view of the designed DOE.

The image of particles acquired using the hybrid lens is presented in Figure 5 (a). One can notice that for this case all particles within the measurement volume are in focus. Their size on the image is almost the same, contrary to the defocused images obtained without using the DOE.
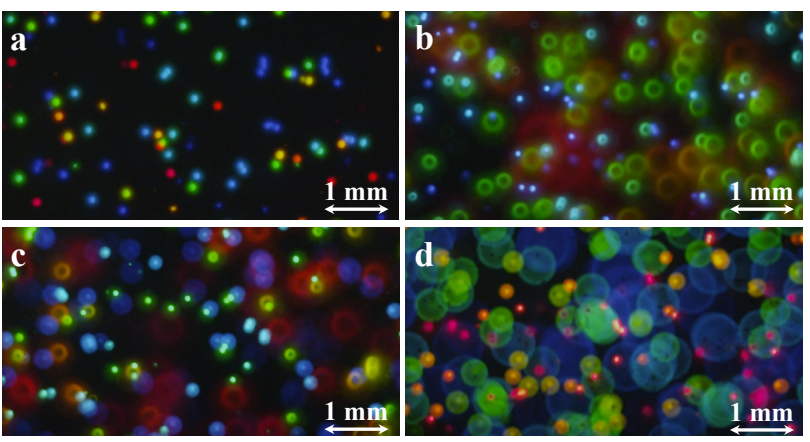

Fig. 5. Comparison of subsections of the images acquired using a hybrid lens and a refractive lens. (a) Image obtained with the hybrid lens (DOE + lens). (b, c, d) Images obtained when using only the refractive lens. The focus is adjusted respectively for blue (b), green (c) and red (d) particles.

Measured flows. Two types of experiments were realized using transparent, rectangular tanks made of glass plates placed on a brass metal support:

- Experiments with a ground truth were performed using a high viscosity transparent fluid (PSF-1,000,000 cSt Pure Silicone Fluid). Its viscosity is one million times higher than that of water. White particles (White Polyethylene Microspheres, with a diameter in the range $[90,106 \mu \mathrm{m}]$ ) were introduced to this liquid. This involved heating the liquid while stirring in the particles, followed by vacuum treatment to eliminate bubbles. After cooling the liquid, the particles become frozen in place. Then, experiments were conducted by applying a known movement (translations or rotation) to the tank using micro-meter stages. Therefore, the particle motion is known, since they are immobile with respect to the tank.

- Experiment without "ground truth" were realized using the same particles, after introducing them in a tank containing tap water. A small amount of surfactant (Polysorbate 80 Water) is added in order to reduce the surface tension of water. This is to avoid the agglomeration of particles in the tank. In this case, the particle motion is generated manually through stirring, pouring, and similar excitations.

\subsection{Velocity Vector Field Reconstruction Results}

In this section, we first evaluate our proposed approaches based on synthetic examples for ground truth comparisons. Then, we conduct two types of experiments, where the first one is to move particles with known motion vector, verifying the accuracy of our methods on real data, the second one is to work on practical fluids.

Synthetic simulations. To quantitatively assess our reconstruction method, we tested our algorithm on simulated data. A volume with the size of $100 \times 100 \times 20(X \times Y \times Z)$ was simulated and we randomly generated 1000 particles in the volume. The particles were advected over time by ground truth flow vectors that were generated using the method of Stam [1999], such that we can obtain time evolved particle distributions from a forward simulation that is completely decoupled from our implementation. Using the image 
formation model from Equation 1, we simulated a time sequence of 5 captured images.

We compare our proposed velocity vector reconstruction algorithm, referred to "S-T div-H\&S", with the general multi-scale HornSchunck algorithm "H\&S" [Meinhardt-Llopis et al. 2013] and its extension by introducing divergence free constraint as a proximal operator "div-H\&S" [Gregson et al. 2014]. Note that the last two approaches compute the motion between one pair of frames independently, while our approach works on a sequence of frames simultaneously.
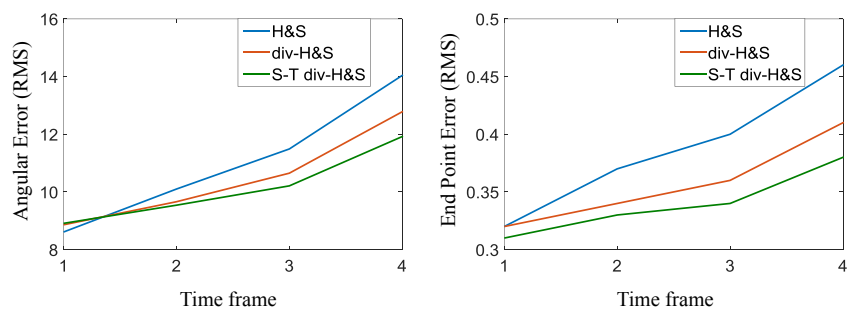

Fig. 6. Numerical comparisons with ground truth data for different algorithms. Left: Average angular error (in degrees). Right: Average end-point error (in pixels).

For evaluation, we use two metrics known from the optical flow literature: the average end-point error, i.e. the average Euclidean distance of the true and estimated particle positions after advection, and the average angular error, i.e. the average discrepancy in flow direction. In Figure 6 we show how both types of error accumulate over multiple frames, which is a good indicator for the accuracy of path lines generated through forward integration in the estimated velocity fields. As expected, the reconstruction errors increase over time in all methods. However, by considering temporal coherence, our proposed method exhibits better performance compared to the other two approaches. We point out that a temporal smoothness regularizer may not necessarily result in improved reconstruction results at each particular time step, however, it conveys better estimations in the temporal domain. This is essential for video frames captured in real-world experiments.

We also ran experiments on simple analytical flows, specifically one vortex with the rotation axis aligned with the optical axis, and one with the rotation axis orthogonal to the optical axis. The results (shown Figure 7) are consistent with the above results on more complex simulated flows. In the first case, the mean of the average end-point error for a sequence of 5 frames are $0.54,0.52$ and 0.49 in pixels, and the mean of the average angular error are 8.06, 7.77 and 7.08 in degrees respectively for "H\&S", "div-H\&S" and "S-T div-H\&S" approaches. As for the latter one, the mean of the average end-point error are $0.79,0.77$ and 0.73 in pixels, and the mean of the average angular error are 17.15, 16.24 and 13.65 in degrees. These results verify that the temporal smoothness term truly boosts the overall reconstruction results for a sequence of frames. Moreover, we could observe a better estimation for flows in the longitudinal plane than those in the transverse plane. This will be further discussed in the following section.
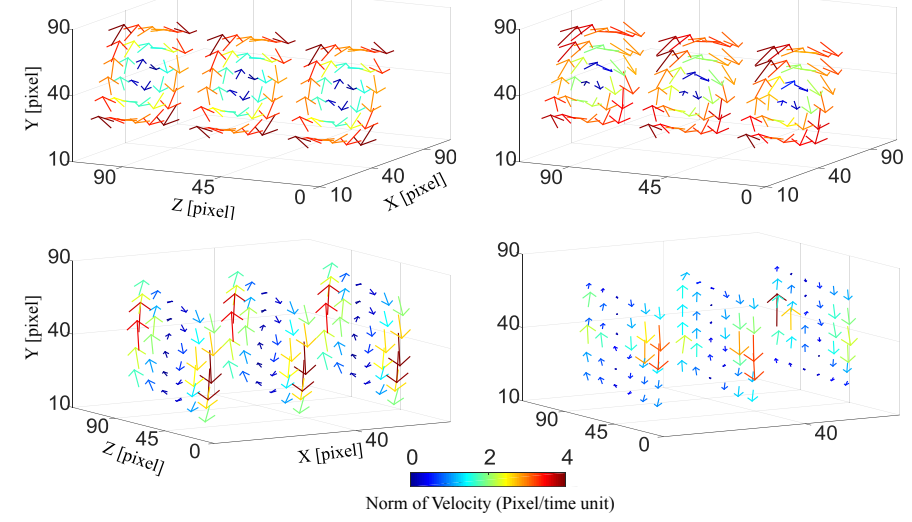

Fig. 7. Ground truth (left) and reconstructed (right) results for simple analytical flows. Top: Rotation around axis aligned with the optical axis. Bottom: Rotation around axis orthogonal to optical axis.

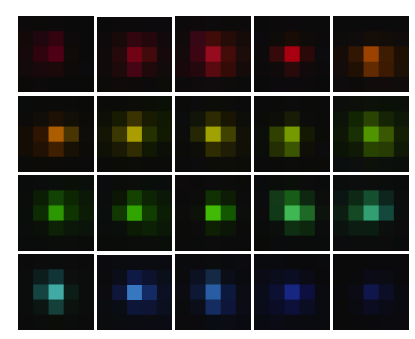

Fig. 8. Calibrated PSFs for different layers along depth direction. From near camera side to far-end of the camera.

Experiments with a ground truth. To evaluate the effectiveness of our proposed methods on real captured data, we firstly conduct the experiments with a tank containing seeded particles in high viscosity liquid. The tank is put on a multi-dimensional translation/rotation stage such that reconstruction results of the algorithm can be compared with ground truth movements. Three independent tests are performed:

(1) Translation in the $x$ direction (i.e. perpendicular to camera line of sight): 5 frames were acquired. Between each two successive frames, a translation of $0.2 \mathrm{~mm}$ in the $x$ direction was applied.

(2) Translation in the $z$ direction (i.e. along the camera line of sight): 5 frames were acquired. Between each two successive frames, a translation of $0.5 \mathrm{~mm}$ in the $z$ direction was applied. In this case, the translation is larger, in order to observe easily the color change.

(3) An approximation of rotation around the vertical ( $y$ ) axis in a clockwise direction. With our setup of "frozen" particles in a volume, only an approximation of this rotational motion is possible, since it is not possible to tilt the tank relative to the camera line of sight to avoid distorting the volume by refraction. We therefore approximate rotational flow by rotating the rainbow illumination pattern relative to 

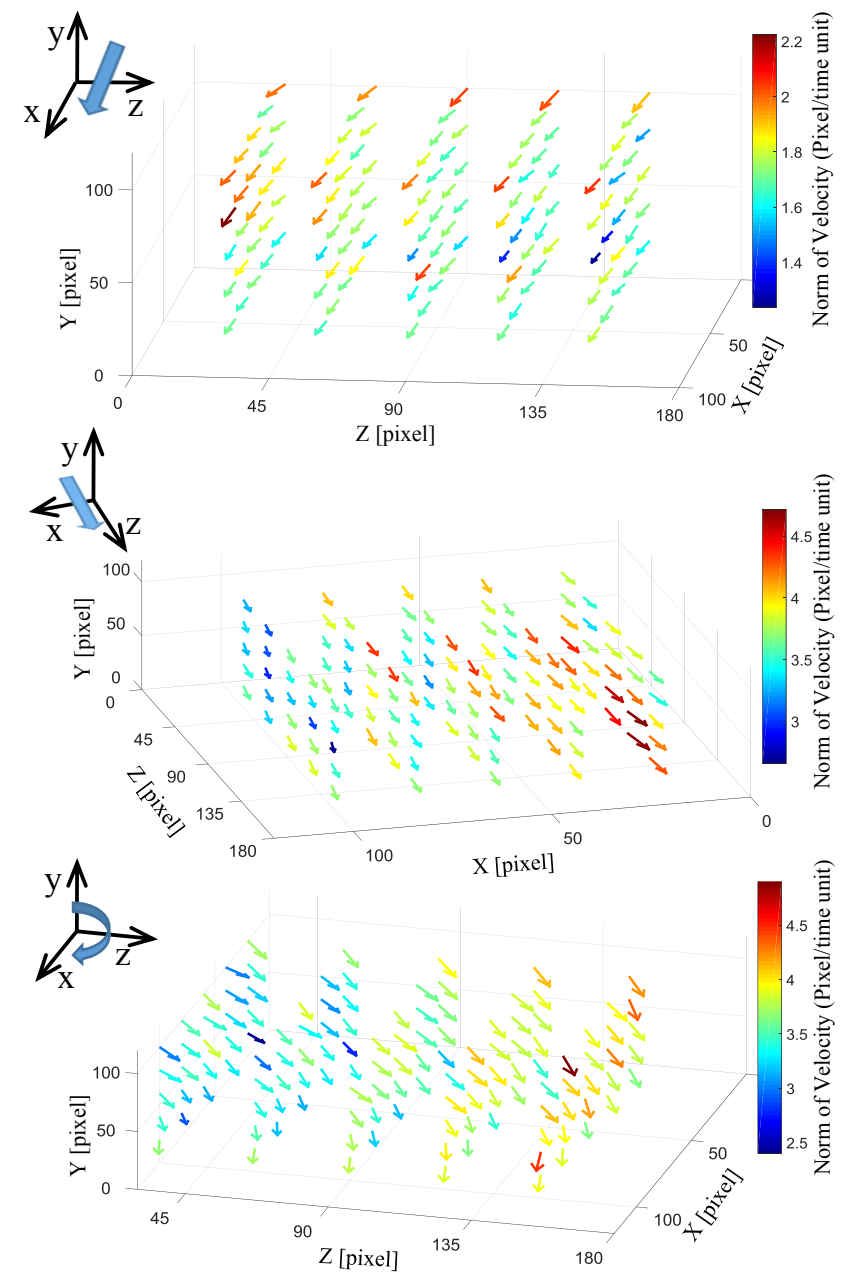

Fig. 9. The reconstructed velocity vector fields induced by moving the measurement volume with a rotation stage. Top: Translation along $x$ direction. Middle: Translation along $z$ direction (towards the camera). Bottom: Rotate along y axis in clockwise direction. The magnitude of the vectors are coded by color.

the tank. In practice, the tank and the camera are mounted together on a rotation table with fixed relative position, and the lighting setup is fixed. The rotations were performed from an angle of $-8^{\circ}$ to $8^{\circ}$ (the reference is defined when the tank is aligned with the (x,z) directions). Between each two successive frames, a rotation of an angle equal to $4^{\circ}$ was applied.

Before processing the captured images, we first pass them through a Gaussian filter and then downsample them by a factor of 8 , hence the resolution for the downsampled image is about $100 \mu \mathrm{m} /$ pixel, approximately one particle per image pixel. We discretize the wavelength coordinate into 20 levels, corresponding to $900 \mu \mathrm{m} /$ layer. The calibrated point spread functions for each levels are shown in Figure 8. It should be noted that the resolution along the wavelength coordinate is about 9 times coarser than that in $x-y$ plane.

The reconstructed velocity vector fields are visualized in Figure 9. The overall structures of the reconstructed flow in all three cases reveal that a significant part of the real flow structures are reproduced.

Furthermore, we can numerically analyze the reconstructed results with respect to the ground truth movements. In the experiments, the $x$-axis and the $z$-axis translations move respectively $200 \mu \mathrm{m}$ and $500 \mu \mathrm{m}$ in one time step, which corresponds in the captured images to 2 and 5 pixels. In the rotation test, the total rotation $\frac{\pi}{45} \mathrm{rad}$, and the 2D plane of the test section has the physical size of $10 \mathrm{~mm} \times 18 \mathrm{~mm}(x \times z)$, and distance from the center of the test section to the center of the disk is $10 \mathrm{~mm}$, hence the practical magnitudes of the displacements are about $334 \mu \mathrm{m}$ (3.3 pixel sizes) for the part at the near-end of the disk center and $506 \mu \mathrm{m}$ (5.1 pixel sizes) for the part at the far-end of the disk center. The computed magnitudes of the flow vectors are encoded by color in our represented results.

The mean of the norm of the velocity in the left translational experiment is 1.75 pixel sizes with standard deviation of 0.15 , while the mean of that in the experiment of translating towards camera is 3.48 pixel sizes with standard deviation of 0.79 . We can see that reconstructed flow vectors reveal higher accuracy for the flow perpendicular to the optical axis with respect to the flow in longitudinal direction. This is reasonable since: (1) depth resolution is highly limited compared to lateral resolution as camera is much more sensitive to the spatial change of objects in 2D plane than the change of wavelength, which results in coarser reconstructed flow vectors along the wavelength coordinate. (2) the error may also come from a bias of reconstructed particle distributions. Determination of the spatial positions of the particles along $z$ axis involves higher uncertainties. Moreover, distortion caused by the refractive effect of the applied high viscosity materials, arises when moving the tank along the $z$ axis. As the thickness of the material between camera and illuminated particles changes, the PSFs are altered simultaneously. Fortunately, this issue does not exist when measuring practical fluid flow, where the particles move, instead of the light beam. Though facing the fact of relatively low reconstruction accuracy for flow in axial direction, not only flow in simple translational structures, but also vortical flows are reasonably reproduced, and the error in wavelength axis is within a certain tolerance, which, in general, is no more than half of length of the discretization intervals.

Experiments without ground truth. Finally, we test our RainbowPIV system on four different real flows of varying complexity (Figures 10-13). Using the setup described in Section 4.1, we captured image sequences of fluids at a frame rate of $30 \mathrm{~Hz}$, and downsampled the images by a factor of 8 from an original resolution of $4096 \times 2160$ to $512 \times 270$. The wavelength coordinate was discretized into 20 levels (10nm/level), hence the maximum grid resolution for any experiment was reach $512 \times 270 \times 20$, although additional cropping was performed on some datasets to only reconstruct regions with interesting flows. The voxel pitch in the $(x, y)$ plane is $100 \mu m$, while along the $z$ axis it is $900 \mu m$. 


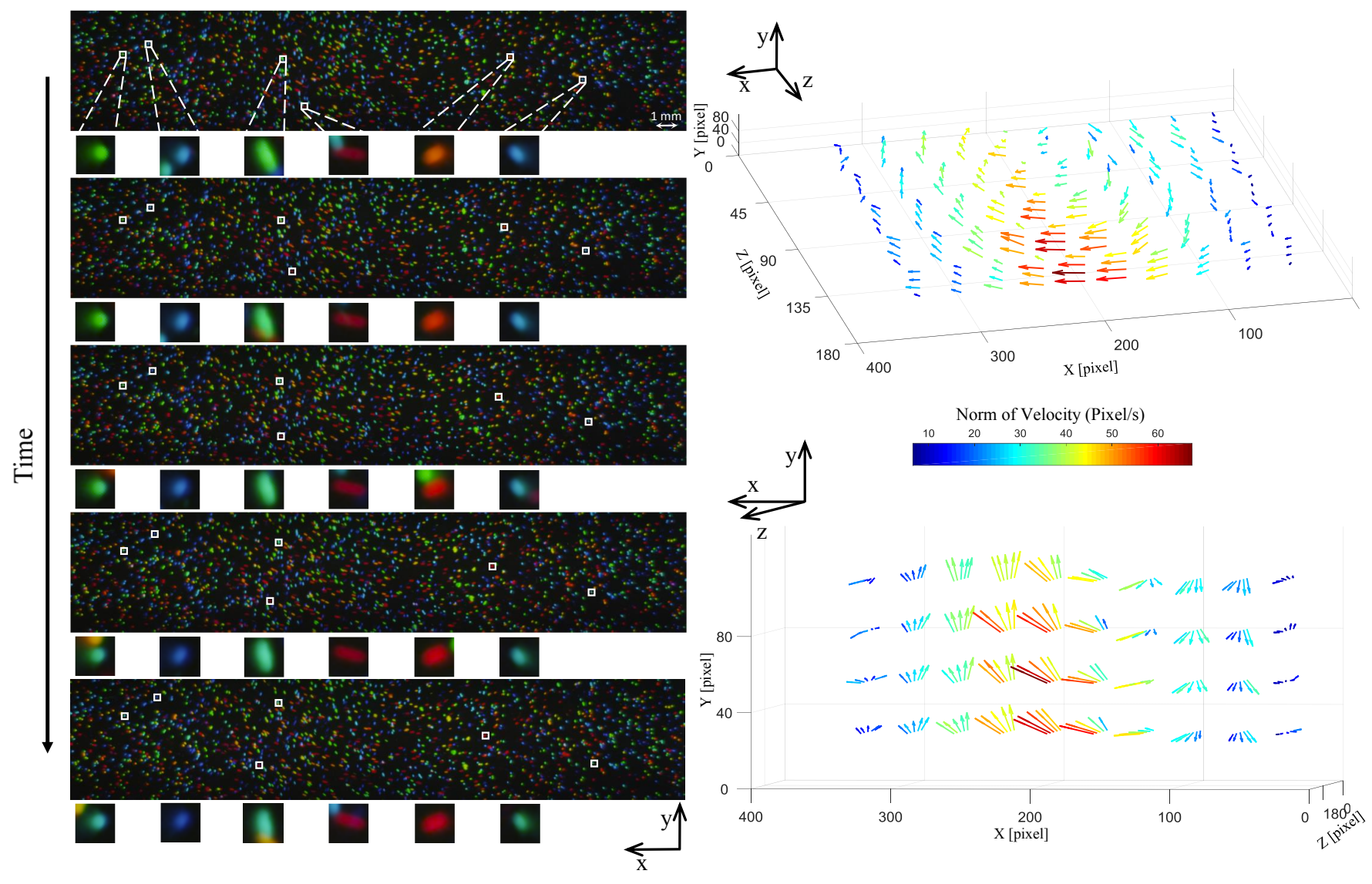

Fig. 10. Left: 5 successively captured images (without post-processing) in a video frame. Six representative particles are tracked in the time sequential frames to verify the reconstructed flow structure. Right: Computed flow vectors according to the given frame data, viewing from different angles. Please see supplemental video for better visualization.

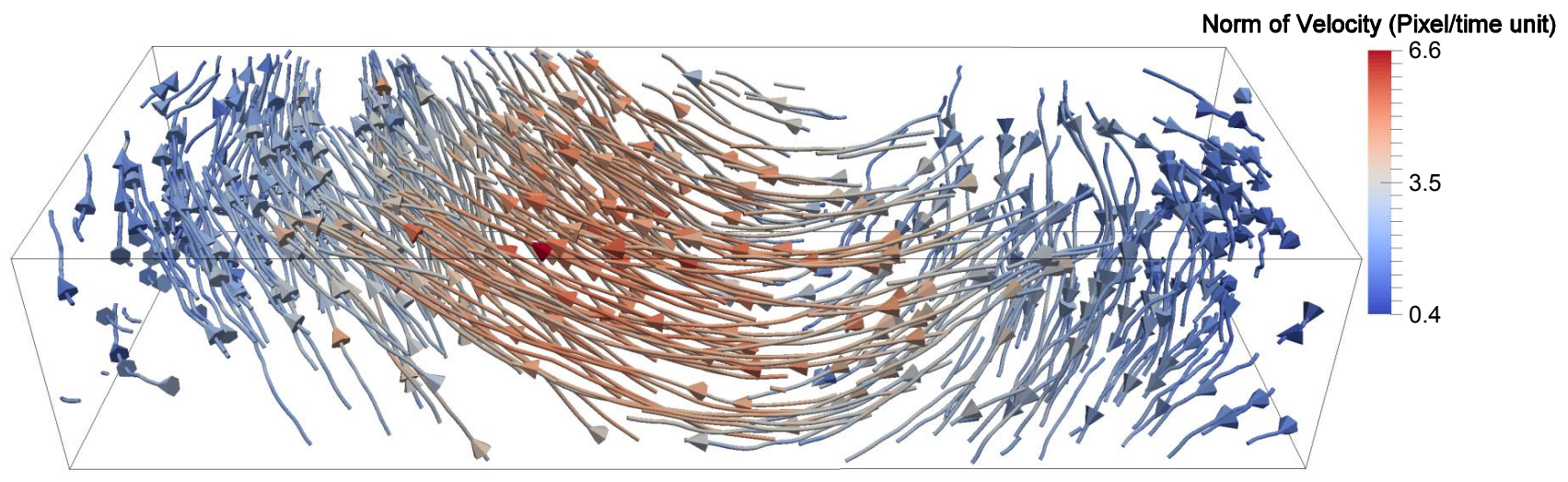

Fig. 11. Path line visualization of the dataset from Figure 10.

The parameters for the optimization method were kept the same for all datasets $\left(\kappa_{1}=\kappa_{2}=0.01, \kappa_{3}=10^{-5}, \kappa_{4}=10^{-7}\right)$. Only two outer loops were required for all datasets, with $30-50$ iterations in the inner loop of the position estimation subproblem, two inner loops for the velocity estimation problem, and finally five loops for each frame within each velocity estimation step. The reconstruction time for the largest dataset was 125 minutes on a $2.50 \mathrm{Ghz}$ Intel Xeon E5-2680 CPU with 128GB RAM. Roughly $1 / 3$ of that time was spent 


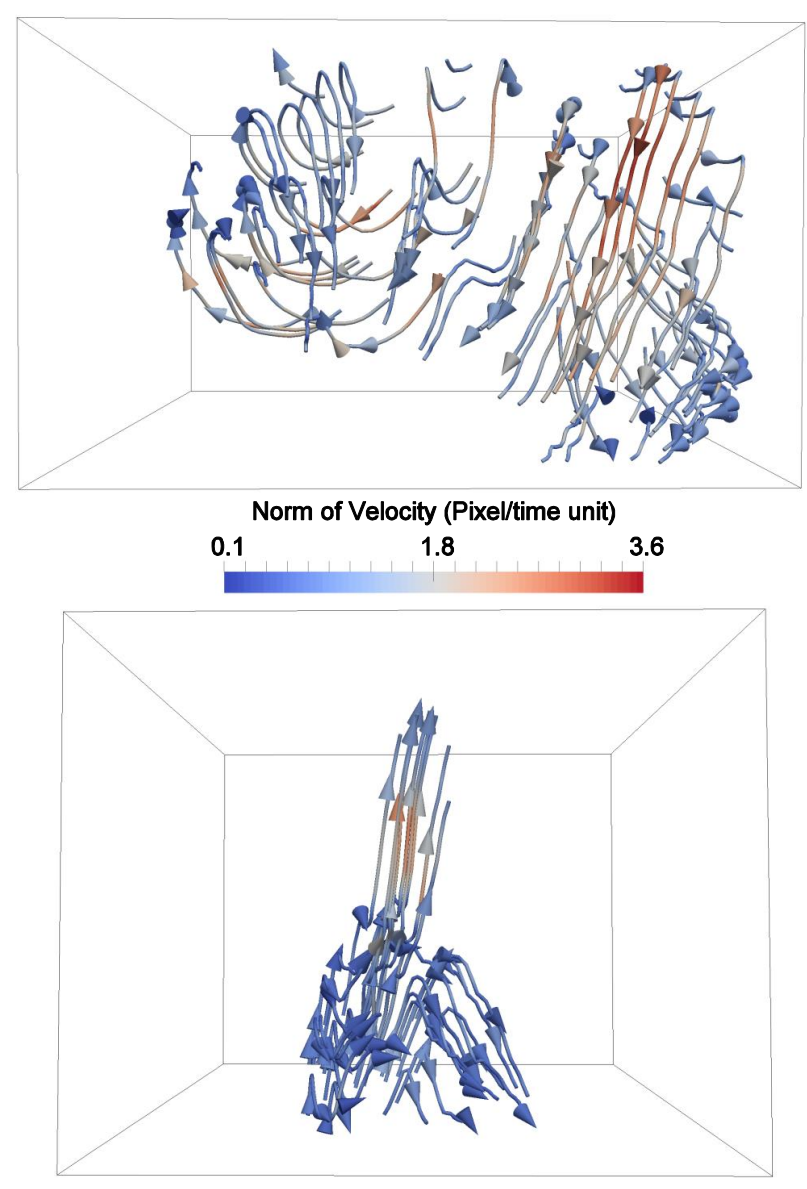

Fig. 12. Path line visualization for two more datasets, corresponding to a drop of water being dripped into the volume from the top (top image), and a small jet of water being injected from the bottom (bottom image).

on the position estimation, and the remaining $2 / 3$ on the velocity estimation.

Five successive captured images are shown in the left hand side of Figure 10, and the reconstructed velocity vectors are visualized on the right hand side of the same figure. Six representative particles are manually selected to verify the accuracy of the computed flow vectors. The first particle moves upward in the image plane, and the color of it changes from green to cyan, which states that it moves away from the camera. The second particle moves upward and slightly to the right in the image plane and in the depth direction, it moves to the far-end of the camera. The third particle moves to the upper left, color changes from green to cyan. The fourth particle quickly moves to the left hand side with no significant color change. From the fourth particle, we can observe a certain amount of motion blur due to its large velocity. The fifth and sixth particle move downwards in the image plane and towards camera in the wavelength domain, while the orange one moves to the right and blue one moves to the left. Comparing the motion of these chosen particles with the corresponding flow vectors in the reconstructed results, it reveals that overall agreement is achieved. In addition, the actual stirred flow structure is supposed to be a vortex, rotating in a clockwise direction. We observe that the key features of the vortex structure are well reconstructed by our developed methods. A path line visualization of the same velocity data is shown in Figure 11. Note that the particles in the visualization are seeded synthetically and do not directly correspond to RainbowPIV particles. Please also refer to the supplemental video for dynamic visualizations of all results.

Figure 12 shows two more data sets, one with a drop of water being dripped into the volume from the top, and one where a small amount of liquid is injected into the volume form the bottom. The recovered flow field in both cases is consistent with both the expectations and the observed RainbowPIV frames (see video).

Finally, the most complex example is shown in Figure 13. This flow was generated by strongly stirring the fluid, and then letting it set. After a while, the pictured two-vortex structure can be observed. Like many fluid imaging methods, RainbowPIV has problems reconstructing flows with strong motion blur. This limits our ability to reconstruct the early stages of this experiment. To overcome this limitation, high speed cameras could be used in conjunction with stronger light sources.

\section{CONCLUSION}

We have introduced a novel RainbowPIV system coupled with optimization strategies, which enables us to recover the 3D fluid flow structures using a single color camera, greatly reducing the hardware setup requirements and easing calibration complexity compared to the other approaches handling 3D-3C measurements. Our approach is implemented by illuminating particles in the volume with "rainbow" light such that the depth information of the particles is color-coded into the captured images, and the 3D trajectory of particles can be tracked by analyzing the $2 \mathrm{D}$ spatial motion in the image plane and the color change in the wavelength domain. A specially designed DOE helps to focus all the wavelength planes on the sensor plane simultaneously, to achieve high lateral resolution and relatively large depth of focus at the same time. We then formulate an inverse problem to reconstruct the particle positions in 3D using a sequence of frames to alleviate the ambiguity issues of identifying particle positions from a single frame. With the recovered particle locations at different time steps, a further step is taken to reconstruct the fluid velocity vector fields. An optimization problem integrating the conventional Horn-Schunck algorithm with physical constraints is proposed to compute the flow vectors.

We demonstrate our approach both on synthetic flows induced by moving a frozen particle volume and by using a real stirred flow. Overall, our method can robustly reconstruct a significant part of the flow structures at good accuracy.

The primary drawback of our system is the limited spatial resolution along the wavelength (depth) coordinate. Due to the existence of noise and light scattering issues, and relatively low sensitivity of the camera to the wavelength change, at current stage the wavelength coordinate is not allowed to be discretized any further. In the future this situation could be improved by making use of the IR end of the spectrum instead of blue light, where camera sensitivity is rather low. Other possible improvements include the use of cameras 


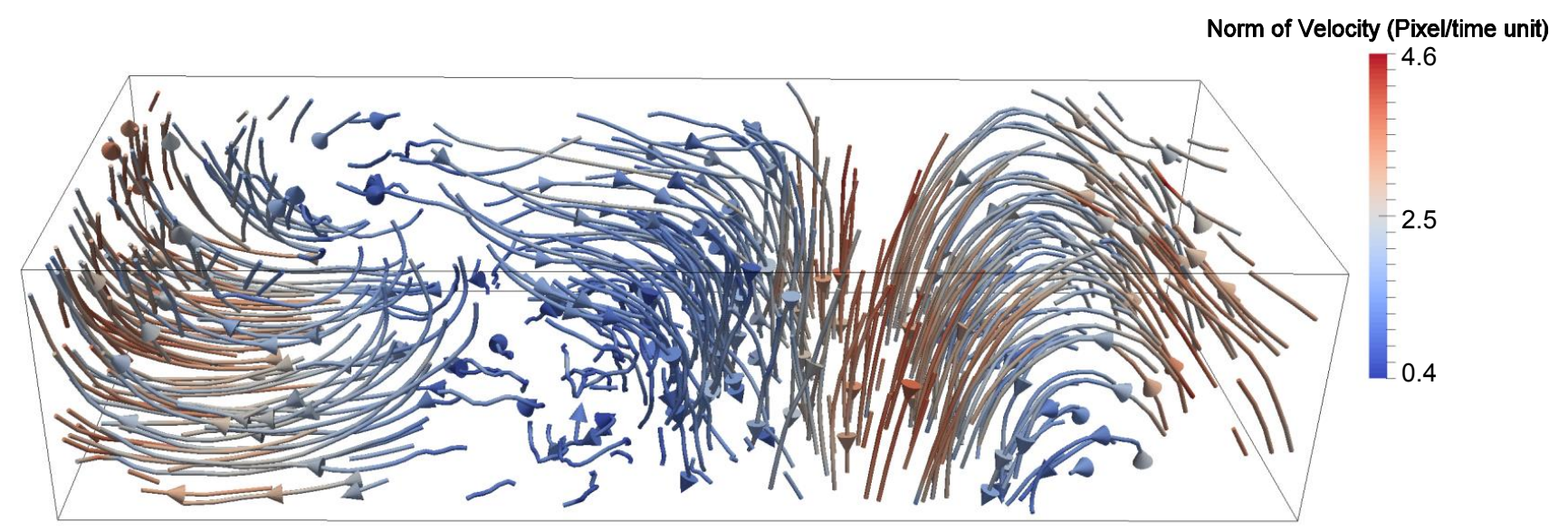

Fig. 13. Path line visualization of a complex flow created by stirring the fluid. Note the two vortices that interact in a complex fashion. The visualization uses virtual particles that do not correspond directly to real particles imaged with RainbowPIV.

with additional color primaries, or primaries that are optimized for this task.

Furthermore, our current system can only measure velocities within a flow volume of fixed dimensions, which are determined in the axial direction by the wavelength spread of the generated rainbow volume and the matching chromatic aberration in the DOE camera optics. In the future we intend to address this issue by designing a dynamically reconfigurable rainbow light engine. In addition to having an adjustable depth range, this Rainbow light engine will use diffractive optics to provide better light efficiency than the currently used linear filter. On the camera side, a viable solution already exists in the form of encoded diffractive optics [Heide et al. 2016], which allows for diffractive lenses with dynamically adjustable focal length. However, integration of the encoded DOE and the new light engine into a new RainbowPIV setup requires still a significant amount of system development.

Despite these current limitations, on account of the simple setup and good accuracy, our system can be easily implemented and applied to investigate new types of fluid flows in the future.

\section{A APPENDIX}

Proximal operators for Algorithm 1 and 2

This section describes the derivation of proximal operators in Algorithm 1 and 2 . To simplify the notations, we denote $\mathbf{z}^{j}-\mathbf{q}^{j}$ as $\mathbf{d}^{j}$, $\mathbf{p}^{j+1}+\mathbf{q}^{j}$ as $\mathbf{e}^{j}, \mathbf{u}_{t}^{j+1}+\mathbf{q}^{j}$ as $\mathbf{h}^{j}$, and $\mathbf{p}_{t+1}\left(\mathbf{u}_{t},-\Delta t\right)$ as $\hat{\mathbf{p}}_{t+1}^{-}$.

For Algorithm 1:

$$
\begin{aligned}
& \mathbf{p}=\operatorname{prox}_{\sigma_{1} F_{1}}(\mathbf{d}) \Rightarrow \\
& {\left[\begin{array}{c}
\sigma_{1}\left(\mathbf{A}^{T}\left(\mathbf{A} \mathbf{p}_{1}\right)+f_{1,2}(\mathbf{p})\right)+\mathbf{p}_{1} \\
\cdot \\
\sigma_{1}\left(\mathbf{A}^{T}\left(\mathbf{A p}_{t}\right)+f_{t, t-1}+f_{t, t+1}\right)+\mathbf{p}_{t} \\
\cdot \\
\cdot \\
\sigma_{1}\left(\mathbf{A}^{T}\left(\mathbf{A p}_{T}\right)+f_{T, T-1}\right)+\mathbf{p}_{T}
\end{array}\right]=\left[\begin{array}{c}
\sigma_{1} \mathbf{A}^{T} \mathbf{i}_{1}+\mathbf{d}_{1}^{j} \\
\cdot \\
\cdot \\
\sigma_{1} \mathbf{A}^{T} \mathbf{i}_{t}+\mathbf{d}_{t}^{j} \\
\cdot \\
\cdot \\
\sigma_{1} \mathbf{A}^{T} \dot{\mathbf{i}}_{T}+\mathbf{d}_{T}^{j}
\end{array}\right]}
\end{aligned}
$$

where

$$
\begin{aligned}
& f_{t, t+1}(\mathbf{p})=2 \kappa_{2} \mathbf{p}_{t} \odot\left(\mathbf{p}_{t}-\hat{\mathbf{p}}_{t+1}^{-}\right)+\kappa_{2}\left(\mathbf{p}_{t}-\hat{\mathbf{p}}_{t+1}^{-}\right)^{\circ 2}, \\
& f_{t, t-1}(\mathbf{p})=2 \kappa_{2} \mathbf{p}_{t-1} \odot\left(\hat{\mathbf{p}}_{t}^{-}-\mathbf{p}_{t-1}\right) . \\
& \quad \mathbf{z}=\operatorname{prox}_{\tau_{1} H_{1}}\left(\mathbf{e}^{j}\right) \Leftrightarrow \\
& \mathbf{z}=\Pi_{[0,1]}\left(\left(\mathbf{e}^{j}-\tau_{1} \kappa_{1} \mathbf{w}\right)_{+}-\left(-\mathbf{e}^{j}-\tau_{1} \kappa_{1} \mathbf{w}\right)_{+}\right) .
\end{aligned}
$$

In the first term, $\mathrm{p}$ is represented by procedural operator on the left hand side of Equation 15, and it is solved by Conjugate Gradients. The second term is the point-wise shrinkage operation followed by a projection onto the domain of $[0,1]$.

For Algorithm 2:

$$
\begin{aligned}
\mathbf{u}_{t}= & \operatorname{prox}_{\sigma_{2} F_{2}}(\mathbf{d}) \Leftrightarrow\left(\sigma_{2} \mathbf{A}+\mathbb{I}\right) \mathbf{u}_{t}=\mathbf{d}^{j}-\sigma_{2} \mathbf{b} \\
\mathbf{A}= & \mathbf{p}_{\mathbf{t}} \odot\left(\nabla \hat{\mathbf{p}}_{t+1}^{-}\right)^{\circ 2}+\kappa_{3} \nabla^{2}+2 \kappa_{4} \mathbf{M} \\
\mathbf{b}= & \mathbf{p}_{\mathbf{t}} \odot\left(\left(\hat{\mathbf{p}}_{t+1}^{-}-\mathbf{p}_{\mathbf{t}}\right)-\nabla \hat{\mathbf{p}}_{t+1}^{-} \mathbf{u}_{t}^{k}\right) \nabla \hat{\mathbf{p}}_{t+1}^{-} \\
& -\kappa_{4} \mathbf{M}\left(\Pi_{C_{D I V}}\left(\hat{\mathbf{u}}_{t-1}^{k+}\right)+\Pi_{C_{D I V}}\left(\hat{\mathbf{u}}_{t+1}^{k-}\right)\right),
\end{aligned}
$$

where

$$
\begin{gathered}
\hat{\mathbf{u}}_{t+1}^{k-}=\mathbf{u}_{t+1}\left(\mathbf{u}_{t}^{k},-\Delta t\right), \\
\hat{\mathbf{u}}_{t-1}^{k+}=\mathbf{u}_{t-1}\left(\mathbf{u}_{t-1}^{k}, \Delta t\right) . \\
\mathbf{z}=\operatorname{prox}_{\tau_{2} H_{2}}\left(\mathbf{h}^{j}\right) \Leftrightarrow \mathbf{z}=\Pi_{C_{D I V}}\left(\mathbf{h}^{j}\right) .
\end{gathered}
$$

By applying the fixed-point theorem to tackle the nonlinear optimization problem, $\mathbf{u}^{k}$ in the first term refers to the result in the $k^{t h}$ iteration. We use Conjugate Gradients to solve this linear system in combination with an incomplete Cholesky factorization. The second term is a simple pressure projection step.

Design of the hybrid refractive-diffractive lens

With a standard refractive lens, the thickness of the measurement volume that is in focus is extremely small for many fluid imaging problems of practical interest. In our case, since the wavelength of the light that illuminates the particles varies linearly in the volume, 
the in-focus measurement volume can be easily extended by adding a DOE to the camera optics.

When designing the DOE we must ensure that all wavelengths are focused on the same sensor plane. Moreover, the aperture and the magnification of the hybrid lens should allow for an image of good quality.

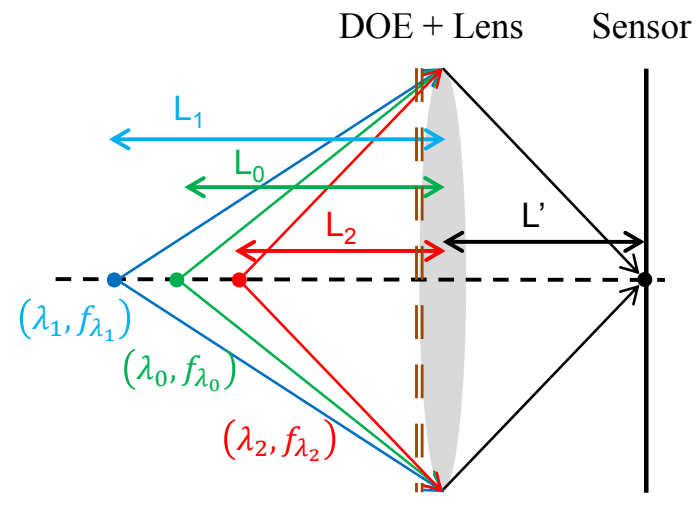

Fig. 14. Schema for hybrid refractive-diffractive lens. All particles illuminated by a light, which wavelength is included in $\left[\lambda_{1}, \lambda_{2}\right]$, will be focused on the sensor plane.

The Figure 14 shows a schematic for a hybrid refractive-diffractive lens. The aim is to design a DOE, that allows us to have all particles illuminated by a light, which wavelength is included in $\left[\lambda_{1}, \lambda_{2}\right]$ focused on the same sensor plane.

A DOE is characterized by its phase, which can be expressed as follows:

$$
\phi(r)=\frac{2 \pi}{\lambda} \cdot \frac{r^{2}}{f_{\lambda}^{D O E}},
$$

where $r$ is the radial distance to the center of the DOE, $\lambda$ is a given wavelength, and $f_{\lambda}^{D O E}$ is the focal length of the DOE associated to the wavelength $\lambda$. Indeed, for a DOE the focal length is spectraldependent, and obeys the following relationship:

$$
\lambda \cdot f_{\lambda}^{D O E}=\text { constant } .
$$

Thereafter, the wavelength $\lambda_{0}=\frac{2 \cdot \lambda_{1} \cdot \lambda_{2}}{\lambda_{1}+\lambda_{2}}$ will be used to design the DOE. Thus, we only need to determine $f_{\lambda_{0}}^{D O E}$, in order to recover the phase of the DOE.

On one hand, the thickness of the measurement volume $\Delta z=$ $L_{1}-L_{2}$ is enforced by the used setup. On the other hand, the distances $L_{1}$ and $L_{2}$ are expressed as follows, as a function of $L^{\prime}$ the distance between the hybrid lens and the sensor, and $f_{\lambda}$ the focal length of the hybrid lens:

$$
L_{1 / 2}=\frac{L^{\prime} \cdot f_{\lambda_{1 / 2}}}{L^{\prime}-f_{\lambda_{1 / 2}}}
$$

Furthermore, $\left(f_{\lambda}\right)$ can be expressed as:

$$
\frac{1}{f_{\lambda}}=\frac{\lambda}{\lambda_{0} \cdot f_{\lambda_{0}}^{D O E}}+\frac{1}{f_{L}} \Leftrightarrow f_{\lambda}=\frac{\lambda_{0} \cdot f_{\lambda_{0}}^{D O E} \cdot f_{L}}{\lambda_{0} \cdot f_{\lambda_{0}}^{D O E}+\lambda \cdot f_{L}} .
$$

By combining the Eqs. 21 and 22 in the expression of $\Delta z$, we obtain:

$$
\begin{aligned}
\Delta z= & \frac{\lambda_{0} f_{\lambda_{0}}^{D O E} f_{L} L^{\prime}}{\left(\lambda_{0} f_{\lambda_{0}}^{D O E}+\lambda_{1} f_{L}\right)-\lambda_{0} f_{\lambda_{0}}^{D O E} f_{L}} \\
& \frac{\lambda_{0} f_{\lambda_{0}}^{D O E} f_{L} L^{\prime}}{\left(\lambda_{0} f_{\lambda_{0}}^{D O E}+\lambda_{2} f_{L}\right)-\lambda_{0} f_{\lambda_{0}}^{D O E} f_{L}} .
\end{aligned}
$$

Here, $f_{L}$ is fixed by the choice of the refractive lens, $\Delta z, \lambda_{1}$ and $\lambda_{2}$ are measured and depend on the illumination setup. Therefore, the focal length of the DOE $f_{\lambda_{0}}^{D O E}$ and the distance $L^{\prime}$ are retrieved by minimizing the difference between the two terms of the equation 23.

To ensure a good quality of the obtained image, we have to add some constraints to this minimization. These constraints involve the aperture $\left(F \#=\frac{L^{\prime}}{D}\right)$ and the magnification $\left(\gamma(\lambda)=\frac{L_{\lambda}}{L^{\prime}}\right)$ of the hybrid lens. Where $D$ is the diameter of the hybrid lens, and $L_{\lambda}$ is the distance between the hybrid lens and the plane illuminated by a light with a wavelength equal to $\lambda$. The constraint on the aperture will improve the signal to noise ratio of the obtained image; while the constraint on the magnification will warrant a good size match between the measurement volume and the acquired image.

Once the optimal focal length $f_{\lambda_{0}}^{D O E}$ retrieved, the DOE is completely characterized by its phase (see Eq. 19).

\section{ACKNOWLEDGMENTS}

The authors would like to thank Thomas Theussl from the KAUST Visualization Core Lab for help with the initial flow visualizations.

\section{REFERENCES}

R.J. Adrian and J. Westerweel. 2011. Particle image velocimetry. Cambridge University Press.

B. Atcheson, I. Ihrke, W. Heidrich, A. Tevs, D. Bradley, M. Magnor, and H-P. Seidel 2008. Time-resolved 3D Capture of Non-stationary Gas Flows. ACM Trans. Graph. 27, 5 (2008), 132

I. Barbu, C. Herzet, and E. Mémin. 2013. Joint estimation of volume and velocity in TomoPIV. In 10TH INTERNATIONAL SYMPOSIUM ON PARTICLE IMAGE VELOCIMETRY-PIV13, 45.

S. Boyd, N. Parikh, E. Chu, B. Peleato, and J. Eckstein. 2011. Distributed optimization and statistical learning via the alternating direction method of multipliers. Foundations and Trends in Machine Learning 3, 1 (2011), 1-122.

E.J. Candes, M.B. Wakin, and S.P. Boyd. 2008. Enhancing sparsity by reweighted âĎs 1 minimization. F. Fourier analysis and applications 14, 5-6 (2008), 877-905.

T.A. Casey, J. Sakakibara, and S.T. Thoroddsen. 2013. Scanning Tomographic Particle Image Velocimetry Applied to a Turbulent Jet. Phys. Fluids 25 (2013), 025102.

G.E. Elsinga, F. Scarano, B. Wieneke, and B.W. van Oudheusden. 2006. Tomographic particle image velocimetry. Experiments in Fluids 41, 6 (2006), 933-947.

R. Fedkiw, J. Stam, and H.W. Jensen. 2001. Visual simulation of smoke. In Proc. ACM Siggraph. 15-22.

N. Foster and D. Metaxas. 1997. Modeling the motion of a hot, turbulent gas. In Proc. ACM Siggraph. 181-188.

J. Gregson, I. Ihrke, N. Thuerey, and W. Heidrich. 2014. From capture to simulation: connecting forward and inverse problems in fluids. ACM Trans. Graph. 33, 4 (2014), 139.

J. Gregson, M. Krimerman, M.B. Hullin, and W. Heidrich. 2012. Stochastic tomography and its applications in 3D imaging of mixing fluids. ACM Trans. Graph. 31, 4 (2012), $52-1$.

J. Gu, S.K. Nayar, E. Grinspun, P.N. Belhumeur, and R. Ramamoorthi. 2013. Compressive Structured Light for Recovering Inhomogeneous Participating Media. IEEE PAMI 35, 3 (2013), 555-567.

S.W. Hasinoff and K.N. Kutulakos. 2007. Photo-consistent Reconstruction of Semitransparent Scenes by Density-sheet Decomposition. IEEE PAMI 29, 5 (2007), 870-885.

T. Hawkins, P. Einarsson, and P. Debevec. 2005. Acquisition of Time-Varying Participating Media. ACM Trans. Graph. 24, 3 (2005), 812-815. 
F. Heide, Q. Fu, Y. Peng, and W. Heidrich. 2016. Encoded diffractive optics for fullspectrum computational imaging. Scientific Reports 6, 33543 (Sept. 2016), 10.

D. Heitz, P. Héas, E. Mémin, and J. Carlier. 2008. Dynamic consistent correlationvariational approach for robust optical flow estimation. Experiments in fluids 45,4 (2008), 595-608

D. Heitz, E. Mémin, and C. Schnörr. 2010. Variational fluid flow measurements from image sequences: synopsis and perspectives. Experiments in Fluids 48, 3 (2010), 369-393.

I. Herlin, D. Béréziat, N. Mercier, and S. Zhuk. 2012. Divergence-free motion estimation In Proc. ECCV. 15-27.

K.D. Hinsch. 2002. Holographic particle image velocimetry. Measurement Science and Technology 13, 7 (2002), R61.

B.K.P. Horn and B.G. Schunck. 1981. Determining optical flow. Artificial Intelligence 17, 1-3 (1981), 185-203.

I. Ihrke and M. Magnor. 2004. Image-Based Tomographic Reconstruction of Flames. In Proc. SCA. 367-375.

I. Kimura, Y. Kohno, T. Ogasawara, and T. Takamori. 1991. Measurement of three dimensional velocity vectors in a flow field using a color spectrum. Transactions of the Society of Instrument and Control Engineers 27, 7 (1991), 755-761.

M. Levoy, R. Ng, A. Adams, M. Footer, and M. Horowitz. 2006. Light field microscopy ACM Trans. Graph. 25, 3 (2006), 924-934

T. Liu, A. Merat, M.H.M. Makhmalbaf, C. Fajardo, and P. Merati. 2015. Comparison between optical flow and cross-correlation methods for extraction of velocity fields from particle images. Experiments in Fluids 56, 8 (2015), 1-23.

T. Liu and L. Shen. 2008. Fluid flow and optical flow. F. Fluid Mechanics 614 (2008), 253-291.

L.M. Lourenco, A. Krothapalli, and C.A. Smith. 1989. Particle image velocimetry. In Advances in Fluid Mechanics Measurements. Springer, 127-199.

K.P. Lynch, T. Fahringer, and B. Thurow. 2012. Three-dimensional particle image velocimetry using a plenoptic camera. American Institute of Aeronautics and Astronautics (AIAA).

T.J. McGregor, D.J. Spence, and D.W. Coutts. 2007. Laser-based volumetric colour-coded three-dimensional particle velocimetry. Optics and Lasers in Engineering 45, 8 (2007), 882-889.

E. Meinhardt-Llopis, J.S. Pérez, and D. Kondermann. 2013. Horn-schunck optical flow with a multi-scale strategy. Image Processing on line 2013 (2013), 151-172.

R. Ng, M. Levoy, M. Brédif, G. Duval, M. Horowitz, and P. Hanrahan. 2005. Light field photography with a hand-held plenoptic camera. Computer Science Technical Report CSTR 2, 11 (2005), 1-11.

K. Okamoto, S. Nishio, T. Saga, and T. Kobayashi. 2000. Standard images for particleimage velocimetry. Measurement Science and Technology 11, 6 (2000), 685.

N. Parikh, S.P. Boyd, and others. 2014. Proximal Algorithms. Foundations and Trends in Optimization 1, 3 (2014), 127-239.

Y. Peng, Q. Fu, H. Amata, S. Su, F. Heide, and W. Heidrich. 2015. Computational imaging using lightweight diffractive-refractive optics. Optics Express 23, 24 (2015), 31393-31407.

S. Pick and F-O. Lehmann. 2009. Stereoscopic PIV on multiple color-coded light sheets and its application to axial flow in flapping robotic insect wings. Experiments in Fluids 47, 6 (2009), 1009-1023.

A.K. Prasad. 2000. Particle image velocimetry. CURRENT SCIENCE-BANGALORE- 79, 1 (2000), 51-60.

P. Ruhnau, A. Stahl, and C. Schnörr. 2007. Variational estimation of experimental fluid flows with physics-based spatio-temporal regularization. Measurement Science and Technology 18, 3 (2007), 755.

D. Schanz, S. Gesemann, and A. Schröder. 2016. Shake-The-Box: Lagrangian particle tracking at high particle image densities. Experiments in fluids 57, 5 (2016), 1-27.

J. Stam. 1999. Stable fluids. In Proc. ACM Siggraph. 121-128.

M. Stanislas, K. Okamoto, C.J. Kähler, J. Westerweel, and F. Scarano. 2008. Main results of the third international PIV challenge. Experiments in Fluids 45, 1 (2008), 27-71.

H. Wang, M. Liao, Q. Zhang, R. Yang, and G. Turk. 2009. Physically guided liquid surface modeling from videos. ACM Trans. Graph. 28, 3 (2009), 90.

T. Watamura, Y. Tasaka, and Y. Murai. 2013. LCD-projector-based 3D color PTV. Experimental Thermal and Fluid Science 47 (2013), 68-80.

C.E. Willert and M. Gharib. 1992. Three-dimensional particle imaging with a single camera. Experiments in Fluids 12, 6 (1992), 353-358.

J. Yuan, C. Schörr, and G. Steidl. 2007. Simultaneous higher-order optical flow estimation and decomposition. SIAM fournal on Scientific Computing 29, 6 (2007), 2283-2304.

Received January 2017; final version April 2017; accepted May 2017 